\begin{tabular}{|l|l|l} 
Int.J.Curr.Microbiol.App.Sci (2020) 9(2): 2696-2710 \\
$\begin{array}{l}\text { International Journal of Current Microbiology and Applied Sciences } \\
\text { ISSN: 2319-7706 Volume } 9 \text { Number } 2(2020) \\
\text { Journal homepage: http://www.ijcmas.com }\end{array}$ \\
$\begin{array}{l}\text { EXCELLENT } \\
\text { PUBLISHERS }\end{array}$
\end{tabular}

\title{
Evaluation of single cross hybrids in tomato (Solanum lycopersicum) under IDM and Non-IDM conditions for resistance to bacterial wilt and tomato leaf curl virus diseases
}

\author{
T. L. Dheemanth ${ }^{1 *}$, B. G. Prakash ${ }^{1}$, M. K. Honnabyraiah ${ }^{2}$, \\ A. P. Mallikarjun Gowda ${ }^{3}$ and S. Mohan Kumar ${ }^{1}$ \\ ${ }^{1}$ Department of Biotechnology and Crop Improvement, ${ }^{2}$ Department of Fruit Science, College \\ of Horticulture Kolar, University of Horticultural Sciences Bagalkot - 563 103, India \\ ${ }^{3}$ Krishi Vigyan Kendra, Bengaluru Rural, University of Agricultural Sciences, \\ Bangalore - 561 205, India \\ *Corresponding author
}

\section{Keywords}

Tomato, Bacterial wilt, ToLCV, Solanum lycopersicum

Article Info

Accepted:

20 January 2020

Available Online:

10 February 2020

\section{A B S T R A C T}

Thirteen single cross hybrids were evaluated at Mudhude village, Arsikere taluk, Hassan district, Karnataka during summer 2017 under IDM and Non-IDM conditions with the objective of testing the performance of commercial hybrids and resistance to bacterial wilt and ToLCV diseases. The top three high yielders were Abhinav, Badshah and 1057. The top high yielding hybrid was Abhinav and performed better than other single cross hybrids including both checks with yield of $6.63 \mathrm{~kg}$ per plant with single fruit weight of $87.24 \mathrm{~g}$. It also possessed highest number of fruits per plant (77.53) and recorded lycopene content of $5.98 \mathrm{mg} / 100 \mathrm{~g}$. The second high yielding SCH was Badshah with yield of $5.11 \mathrm{~kg}$ per plant with single fruit weight of $85.47 \mathrm{~g}$ and number of fruits per plant of 62.33. The third high yielding SCH was 1057 which yielded $5.08 \mathrm{~kg}$ per plant with highest single fruit weight of $100.27 \mathrm{~g}$. The top three bacterial wilt resistant single cross hybrids were Abhinav (1.56\%), US3140 (6.77\%), Arka Rakshak (6.77\%) and top three ToLCV resistant single cross hybrids were Abhinav (5.02), Arka Rakshak (10.62), Sonam (11.45).

\section{Introduction}

Tomato (Solanum lycopersicum L.; $2 \mathrm{n}=24$ ) is one of the important and most widely grown versatile vegetable crops of both tropics and sub tropics. It belongs to the family Solanaceae and ranks second in vegetables after potato. Out of many diseases in tomato, bacterial wilt and tomato leaf curl virus (ToLCV) diseases are major diseases devastating crop and farmers income. Bacterial wilt caused by Ralstonia solanacearum (Yabuuchi et al., 1995) has remained a major destructive plant disease 
and leaf curl caused by the Tomato Leaf Curl Virus (ToLCV), a heterogenous complex of whitefly transmitted geminivirus. Hence this study was undertaken with the objective to identify the bacterial wilt and ToLCV resistant single cross hybrids and also their yield potential for Central dry zone of Karnataka.

\section{Materials and Methods}

Thirteen commercial hybrids of tomato which are presently being grown extensively by majority of farmers in Karnataka were used along with checks Arka Rakshak and Arka Samrat from IIHR, Bangalore (Table 1). The thirteen commercial $\mathrm{F}_{1} \mathrm{~s}$ are ruling hybrids.

\section{Nursery for single cross hybrids (parents)}

All thirteen $\mathrm{F}_{1}$ hybrids seeds were treated with capton@0.1\% before sowing to prevent damping-off disease as a precautionary measure, which usually prevalent in kharif season and the seeds were sown in beds of 2 $\mathrm{m}, 1.2 \mathrm{~m}$ and $15 \mathrm{~cm}$ length, width and height respectively on February 2017 during summer season. All nursery package of practices were followed. Thus, the seedlings were raised up to 30 days after sowing.

\section{Layout and transplanting of single cross} hybrids (parents)

Thirty day old seedlings of the thirteen single cross hybrids of tomato were transplanted in main field with spacing of $60 \mathrm{~cm} \times 60 \mathrm{~cm}$ on March 2017 at Mudhude village, Arsikere taluk, Hassan district, Karnataka (Central Dry Zone of Karnataka). The design followed was Randomized Block Design (RBD) in three replications. In each replication, each $F_{1}$ hybrid seedlings were planted in a plot size of $4.8 \mathrm{~m} \times 2.4 \mathrm{~m}$ consisting of four rows with each row having eight plants and thus totally there were thirty two plants per plot. All the recommended package of practices of this zone were followed throughout crop duration for IDM and Non-IDM conditions according to requirement but control measures for disease and pest control were not taken in Non-IDM condition. The crop was given frequent irrigations whenever necessary. Evaluation was done in sick plots where tomato after tomato crops was raised continuously for many years.

The hybrids were evaluated separately in two conditions viz., Integrated disease management (IDM) condition - that is with all chemical and biological control measures against diseases taken to avoid diseases and other one Non-Integrated disease management (Non-IDM) condition - in which no control measures were taken to avoid diseases.

\section{Observations recorded}

Observations on five randomly selected plants in each replication were recorded for various growth, yield and quality attributing traits to understand the performance of tomato hybrids

Bacterial wilt incidence

Incidence of bacterial wilt was recorded as and when wilt was observed and per cent wilt incidence (PWI) was calculated.

Based on the per cent wilt incidence single cross hybrids were categorised into four groups as suggested by Mew and Ho (1976) i.e. 0-20: Resistant; 20-40: Moderately resistant; 40-60: Moderately susceptible; 60100: Susceptible.

ToLCV incidence and severity

Based on the per cent of curling and puckering of leaves, the plants were scored using 0-4 scale as suggested by Banerjee and Kalloo (1987) i.e. 0: Symptoms absent; 1: Very mild curling (Upto 25\% leaves); 2: 
Curling and puckering of 26-50\% leaves; 3 : Curling and puckering of 51-75\% leaves; and 4: Severe curling and puckering of $>75 \%$ leaves.

Based on the disease score, per cent disease severity (PDS) was calculated using the following formula:

PDS $=\frac{\text { Sum of numerical rating }}{\text { Total number of plants observed } \times \text { Maximum disease grade }} \times 100$

Based on the per cent disease severity (PDS) and per cent disease incidence (PDI) the coefficient of the infection (CI) was calculated using following formula.

Coefficient of infection $=\frac{\text { PDS } \times \text { PDI }}{100}$

Based on the coefficient of infection the hybrids were categorised into six groups (PDVR, 1997) i.e. 0-4: Highly resistant (HR); 4.1-9: Resistant (R); 9.1-19: Moderately resistant (MR); 19.1-39: Moderately susceptible (MS); 39.1-69: Susceptible (S); 69.1-100: Highly susceptible (HS).

\section{Results and Discussion}

The mean performance of the single cross hybrids for morphological and quality characters under IDM and Non-IDM conditions are presented in Table 2, 3, 4 \& 5 . The results are presented under various subheadings as follows.

\section{Morphological traits}

In IDM condition: The hybrid that flowers or fruits earlier is known to add additional income by exploiting the market demand in the early days of the season, and hence the need is for hybrid with early bearing. Days to first flower varied from 23.8 to 30.2. The minimum days to first flower was observed in Alankar (23.8) followed by Sonam (24.6). Days to first fruit ranged from 30.2 to 35.2.
The minimum days to first fruit was recorded in Sonam (30.2) followed by Alankar (30.33). Number of clusters per plant varied from 14 to 18.87 . Maximum number of clusters per plant was observed in US440 (18.87) followed by Lakshmi (17.47). Number of flowers per cluster ranged from 5.07 to 6.27. Highest number of flowers per cluster was recorded in US3140 (6.27) followed by US440 (6.27). Number of fruits per plant, fruit length, fruit width and single fruit weight are the entities that directly contribute to the yield per plant and thereby to total yield per unit area. Number of fruits per plant varied from 50.8 to 86.33. Maximum number of fruits per plant was observed in Abhinav (86.33) followed by 1057 (76). Single fruit weight ranged from 85.53 to $102.2 \mathrm{~g}$. Highest single fruit weight was recorded in US3140 (102.2 g) followed by 1057 (101.93 g). Fruit length varied from 4.39 to $6.79 \mathrm{~cm}$. Maximum fruit length was observed in 1057 $(6.79 \mathrm{~cm})$ followed by Alankar $(6.31 \mathrm{~cm})$. Fruit width ranged from 4.43 to $5.63 \mathrm{~cm}$. Highest fruit width was recorded in US440 $(5.63 \mathrm{~cm})$ followed by Lakshmi $(5.45 \mathrm{~cm})$. Plant height ranged from 83.87 to $114 \mathrm{~cm}$. Highest plant height was recorded in Badshah $(114 \mathrm{~cm})$ followed by Lakshmi $(112.87 \mathrm{~cm})$. Yield per plant varied from 3.91 to $7.46 \mathrm{~kg}$. Maximum yield per plant was observed in Abhinav (7.46 kg) followed by Ryna (6.48 $\mathrm{kg})$.

In Non-IDM condition: Days to first flower varied from 23.67 to 29.87 . The minimum days to first flower was observed in Alankar (23.67) followed by Sonam (25.27). Days to first fruit ranged from 29.53 to 35.47 . The lowest days to first fruit was recorded in Alankar (29.53) followed by Sonam (30.87). Number of clusters per plant varied from 12.07 to 14.87 . Maximum number of clusters per plant was observed in US440 (14.87) followed by Lakshmi (14.53). Number of flowers per cluster ranged from 3.87 to 5.13. Highest number of flowers per cluster was 
recorded in 1057 (5.13) followed by Abhinav (4.93). Number of fruits per plant varied from 22.2 to 68.73. Maximum number of fruits per plant was observed in Abhinav (68.73) followed by Badshah (54.13). Single fruit weight ranged from 83.27 to $100.73 \mathrm{~g}$. Highest single fruit weight was recorded in US3140 (100.73 g) followed by 1057 (98.6 g). Fruit length varied from 4.31 to $6.35 \mathrm{~cm}$. Maximum fruit length was observed in 1057 $(6.35 \mathrm{~cm})$ followed by Arka Rakshak (6.13 $\mathrm{cm})$. Fruit width ranged from 4.47 to $5.55 \mathrm{~cm}$. Highest fruit width was recorded in 1057 $(5.55 \mathrm{~cm})$ followed by US440 $(5.45 \mathrm{~cm})$. Plant height ranged from 79.6 to $105.47 \mathrm{~cm}$. Highest plant height was recorded in Badshah $(105.47 \mathrm{~cm})$ followed by Lakshmi (105.07 $\mathrm{cm})$. Yield per plant varied from 1.42 to 5.79 $\mathrm{kg}$. Maximum yield per plant was observed in Abhinav $(5.79 \mathrm{~kg})$ followed by Badshah $(4.26$ $\mathrm{kg}$ ). These results were similar to the findings of Arun et al., 2004 and Dheemanth et al., 2017.

Performance of top three high yielding single cross hybrids under both IDM and Non-IDM conditions is presented in Table 6. The top three high yielders were Abhinav $(6.63 \mathrm{~kg}$ ), Badshah $(5.11 \mathrm{~kg})$ and 1057(5.08 kg). In case of IDM and Non-IDM conditions, yield per plant was found to be more in IDM condition compare to Non-IDM condition as no control measures were taken for diseases and pests in case of Non-IDM condition.

\section{Quality traits}

In IDM condition: Shelf life ranged from 23.33 to 32.67 days. Highest shelf life was recorded in 1057 and Badshah (32.67 days) followed by Abhinav (30.33 days). Pericarp thickness varied from 4.67 to $8 \mathrm{~mm}$. Maximum pericarp thickness was observed in US3140 $(8 \mathrm{~mm})$ followed by Arka Samrat $(7.33 \mathrm{~mm})$. Fruits with more number of locules found to be having more juice content in them, whereas fruits with lesser number of locules are said to be physically firmer. Number of locules per fruit ranged from 3 to 4. Highest number of locules per fruit was recorded in Badshah (4) followed by Arka Rakshak (3.33) while the lowest number of locules per fruit was recorded in five SCH's viz., 1005, US3140, Abhinav and Alankar (3). Firmness of fruit is most important trait since it influences the shipping ability and keeping quality of fruit. Firmness varied from 5.33 to $7.1 \mathrm{~kg} / \mathrm{cm}^{2}$. Maximum firmness was observed in 1005 and Shivam $\left(7.1 \mathrm{~kg} / \mathrm{cm}^{2}\right)$ followed by Abhinav $\left(6.9 \mathrm{~kg} / \mathrm{cm}^{2}\right)$. Total soluble solids ranged from 4 to $5.5^{\circ} \mathrm{B}$. Highest total soluble solids was recorded in Lakshmi $\left(5.5{ }^{\circ} \mathrm{B}\right)$ followed by Arka Rakshak $\left(5.17{ }^{\circ} \mathrm{B}\right)$. Lycopene that are known to avoid many types of cancer and also provides attractive red colour to the fruit. Lycopene varied from 4.7 to $6.93 \mathrm{mg} / 100 \mathrm{~g}$. Maximum lycopene was observed in Abhinav (6.93 $\mathrm{mg} / 100 \mathrm{~g})$ followed by Arka Rakshak (6.73 mg/100g).

In Non-IDM condition: Shelf life ranged from 23.33 to 30.33 days. Highest shelf life was recorded in US440 (30.33 days) followed by Alankar (28 days). Pericarp thickness varied from 5.33 to $7.33 \mathrm{~mm}$. Maximum pericarp thickness was observed in Arka Samrat (7.33 $\mathrm{mm})$ followed by Arka Rakshak $(6.67 \mathrm{~mm})$. Number of locules per fruit ranged from 2.67 to 3.67. Highest number of locules per fruit was recorded in Badshah (3.67) followed by Arka Samrat (3.33) while the lowest number of locules per fruit was recorded in US440 (2.67) followed by Ryna, Shivam, Sonam, Abhinav and Arka Rakshak (3). Firmness varied from 4.57 to $7.3 \mathrm{~kg} / \mathrm{cm}^{2}$. Maximum firmness was observed in Abhinav (7.3 $\left.\mathrm{kg} / \mathrm{cm}^{2}\right)$ followed by Ryna $\left(6.6 \mathrm{~kg} / \mathrm{cm}^{2}\right)$. Total soluble solids ranged from 2.5 to $6{ }^{\circ} \mathrm{B}$. Highest total soluble solids was recorded in Abhivan $\left(6{ }^{\circ} \mathrm{B}\right)$ followed by $1057\left(5.17{ }^{\circ} \mathrm{B}\right)$. Lycopene varied from 3.3 to $6.77 \mathrm{mg} / 100 \mathrm{~g}$. Maximum lycopene was observed in Sonam 
(6.77 mg/100g) followed by Arka Samrat $(5.73 \mathrm{mg} / 100 \mathrm{~g})$. These results were similar to the findings of Hazarika and Phookan (2005), Renuka et al., 2014 and Bharathkumar et al., 2017.

\section{Disease reaction of single cross hybrids}

Per cent disease incidence and coefficient of infection was calculated among single cross hybrids for bacterial wilt and ToLCV during summer 2017 under IDM and Non-IDM conditions are presented in Table $7 \& 8$.

In IDM condition: Per cent bacterial wilt incidence ranged from 0 to 25 per cent. Maximum per cent bacterial wilt incidence was recorded in 1005 and Alankar (25\%) followed by Lakshmi (11.11\%), the minimum per cent bacterial wilt incidence was recorded in Abhinav and US440 (0\%) followed by $1057(1.04 \%)$. For ToLCV, coefficient of infection ranged from 0.04 to 6.94. Maximum coefficient of infection was recorded in 1005 (6.94) followed by Badshah (5.62), minimum coefficient of infection was recorded in Abhinav (0.04) followed by 1057 (0.13). The single cross hybrids were classified into four groups based on their reaction to bacterial wilt. Of these, 10 were resistant and 3 were moderately resistant (Table 7). The single cross hybrids were classified into six groups based on their reaction to tomato leaf curl disease. Of these, 9 were highly resistant and 4 were resistant (Table 8).

Table.1 Commercially grown Single Cross Hybrids (SCH) of tomato studied

\begin{tabular}{|c|c|c|}
\hline S1. No. & SCH & Source \\
\hline $\mathbf{1 .}$ & Arka Rakshak* & IIHR \\
\hline $\mathbf{2 .}$ & Arka Samrat* & IIHR \\
\hline $\mathbf{3 .}$ & Alankar & Clause Vegetable seeds. \\
\hline $\mathbf{4 .}$ & Abhinav & Syngenta \\
\hline $\mathbf{5 .}$ & 1057 & Syngenta. \\
\hline $\mathbf{6 .}$ & Badshah & Nunhems \\
\hline $\mathbf{7 .}$ & Lakshmi & Nunhems \\
\hline $\mathbf{8 .}$ & US3140 & US Agri \\
\hline $\mathbf{9 .}$ & US440 & US Agri \\
\hline $\mathbf{1 0 .}$ & 1005 & Ashoka seeds \\
\hline $\mathbf{1 1 .}$ & Sonam & Ashoka seeds \\
\hline $\mathbf{1 2 .}$ & Shivam & Rasi seeds \\
\hline $\mathbf{1 3 .}$ & Ryna & Rasi seeds \\
\hline
\end{tabular}


Table.2 Mean value of single cross hybrids for quantitative characters during summer 2017 under IDM condition

\begin{tabular}{|c|c|c|c|c|c|c|c|c|c|c|c|}
\hline $\begin{array}{l}\text { Sl. } \\
\text { No. }\end{array}$ & SCH & $\begin{array}{c}\text { Plant } \\
\text { height } \\
\text { (cm) }\end{array}$ & $\begin{array}{l}\text { Days } \\
\text { to } 1^{\text {st }} \\
\text { flower }\end{array}$ & $\begin{array}{l}\text { Days } \\
\text { to } 1^{\text {st }} \\
\text { fruit }\end{array}$ & \begin{tabular}{|c|} 
Number \\
of clusters \\
/plant
\end{tabular} & $\begin{array}{l}\text { Number } \\
\text { of flowers } \\
\text { /cluster }\end{array}$ & $\begin{array}{c}\text { Number } \\
\text { of fruits } \\
\text { /plant }\end{array}$ & $\begin{array}{c}\text { Single } \\
\text { fruit } \\
\text { weight (g) }\end{array}$ & $\begin{array}{c}\text { Fruit } \\
\text { length } \\
(\mathrm{cm})\end{array}$ & $\begin{array}{r}\text { Fruit } \\
\text { width } \\
\text { (cm) }\end{array}$ & $\begin{array}{c}\text { Yield } \\
\text { /plant } \\
\text { (kg) }\end{array}$ \\
\hline 1. & Arka Rakshak & 96.40 & 25.47 & 33.33 & 14.53 & 5.53 & 56.80 & 86.33 & 6.25 & 5.31 & 4.65 \\
\hline 2. & Arka Samrat & 86.73 & 26.20 & 33.27 & 15.13 & 5.47 & 55.53 & 93.87 & 5.99 & 4.95 & 4.38 \\
\hline 3. & Alankar & 93.00 & 23.80 & 30.33 & 16.07 & 5.60 & 73.40 & 86.27 & 6.31 & 4.69 & 6.21 \\
\hline 4. & Abhinav & 83.87 & 27.73 & 34.13 & 15.07 & 6.00 & 86.33 & 88.87 & 6.28 & 5.23 & 7.46 \\
\hline 5. & 1057 & 102.27 & 29.33 & 34.87 & 15.33 & 5.53 & 76.00 & 101.93 & 6.79 & 5.39 & 6.39 \\
\hline 6. & Badshah & 114.00 & 27.73 & 34.20 & 16.40 & 5.07 & 70.53 & 85.53 & 5.66 & 4.48 & 5.95 \\
\hline 7. & Lakshmi & 112.87 & 26.67 & 31.93 & 17.47 & 5.87 & 56.73 & 93.00 & 4.46 & 5.45 & 4.51 \\
\hline 8. & US3140 & 104.80 & 30.07 & 34.57 & 16.97 & 6.27 & 70.67 & 102.20 & 4.40 & 5.42 & 5.91 \\
\hline 9. & US440 & 95.60 & 30.20 & 35.20 & 18.87 & 6.27 & 60.67 & 95.07 & 4.39 & 5.63 & 4.87 \\
\hline 10. & 1005 & 95.60 & 27.20 & 32.93 & 14.00 & 6.20 & 50.80 & 87.07 & 5.52 & 4.43 & 3.91 \\
\hline 11. & Sonam & 107.00 & 24.60 & 30.20 & 16.07 & 5.60 & 62.60 & 91.67 & 4.44 & 5.34 & 5.20 \\
\hline 12. & Shivam & 105.73 & 25.60 & 31.33 & 15.73 & 5.60 & 67.00 & 100.13 & 4.47 & 5.45 & 5.61 \\
\hline 13. & Ryna & 104.00 & 28.40 & 33.13 & 15.27 & 5.53 & 75.87 & 86.13 & 5.58 & 4.44 & 6.48 \\
\hline & Mean & 100.14 & 27.15 & 33.03 & 15.92 & 5.73 & 66.38 & 92.16 & 5.43 & 5.09 & 5.50 \\
\hline & SE & 0.59 & 0.12 & 0.22 & 0.31 & 0.14 & 2.13 & 1.18 & 0.07 & 0.05 & 0.23 \\
\hline & CD@ $9 \%$ & 1.72 & 0.35 & 0.63 & 0.91 & 0.41 & 6.20 & 3.43 & 0.20 & 0.15 & 0.66 \\
\hline
\end{tabular}


Table.3 Mean value of single cross hybrids for quantitative characters during summer 2017 under Non-IDM condition

\begin{tabular}{|c|c|c|c|c|c|c|c|c|c|c|c|}
\hline $\begin{array}{l}\text { Sl. } \\
\text { No. }\end{array}$ & SCH & $\begin{array}{c}\text { Plant } \\
\text { height } \\
\text { (cm) }\end{array}$ & $\begin{array}{c}\text { Days } \\
\text { to } 1^{\text {st }} \\
\text { flower }\end{array}$ & $\begin{array}{l}\text { Days } \\
\text { to } 1^{\text {st }} \\
\text { fruit }\end{array}$ & $\begin{array}{c}\text { Number } \\
\text { of } \\
\text { clusters } \\
\text { /plant }\end{array}$ & $\begin{array}{c}\text { Number } \\
\text { of } \\
\text { flowers } \\
\text { /cluster }\end{array}$ & $\begin{array}{c}\text { Number } \\
\text { of fruits } \\
\text { /plant }\end{array}$ & $\begin{array}{c}\text { Single } \\
\text { fruit } \\
\text { weight (g) }\end{array}$ & $\begin{array}{c}\text { Fruit } \\
\text { length } \\
\text { (cm) }\end{array}$ & $\begin{array}{c}\text { Fruit } \\
\text { width } \\
(\mathrm{cm})\end{array}$ & $\begin{array}{c}\text { Yield } \\
\text { /plant } \\
\text { (kg) }\end{array}$ \\
\hline 1. & $\begin{array}{c}\text { Arka } \\
\text { Rakshak }\end{array}$ & 91.47 & 25.40 & 33.20 & 12.60 & 4.60 & 45.93 & 84.93 & 6.13 & 5.23 & 3.42 \\
\hline 2. & Arka Samrat & 84.00 & 26.40 & 33.13 & 12.07 & 4.40 & 38.00 & 89.33 & 6.09 & 4.79 & 2.67 \\
\hline 3. & Alankar & 91.13 & 23.67 & 29.53 & 13.33 & 4.47 & 30.20 & 83.27 & 5.75 & 4.56 & 2.17 \\
\hline 4. & Abhinav & 79.60 & 27.60 & 33.47 & 12.47 & 4.93 & 68.73 & 85.60 & 5.89 & 4.93 & 5.79 \\
\hline 5. & 1057 & 96.13 & 29.67 & 34.87 & 12.60 & 5.13 & 50.47 & 98.60 & 6.35 & 5.55 & 3.76 \\
\hline 6. & Badshah & 105.47 & 28.13 & 34.07 & 13.00 & 3.87 & 54.13 & 85.40 & 5.53 & 4.51 & 4.26 \\
\hline 7. & Lakshmi & 105.07 & 26.47 & 32.53 & 14.53 & 4.53 & 22.20 & 94.07 & 4.37 & 5.39 & 1.42 \\
\hline 8. & US3140 & 94.40 & 29.60 & 34.93 & 13.20 & 4.47 & 50.13 & 100.73 & 4.33 & 5.32 & 3.87 \\
\hline 9. & US440 & 89.20 & 29.87 & 35.47 & 14.87 & 4.60 & 29.87 & 93.27 & 4.41 & 5.45 & 2.15 \\
\hline 10. & 1005 & 89.87 & 26.53 & 31.40 & 13.87 & 4.60 & 33.20 & 84.13 & 5.50 & 4.63 & 2.10 \\
\hline 11. & Sonam & 102.47 & 25.27 & 30.87 & 13.07 & 4.00 & 42.33 & 88.67 & 4.31 & 5.25 & 2.97 \\
\hline 12. & Shivam & 101.07 & 26.07 & 32.00 & 12.33 & 4.40 & 42.67 & 97.33 & 4.40 & 5.37 & 3.00 \\
\hline 13. & Ryna & 100.60 & 29.87 & 35.33 & 13.20 & 4.47 & 47.40 & 84.47 & 5.36 & 4.47 & 3.53 \\
\hline & Mean & 94.65 & 27.27 & 33.14 & 13.16 & 4.50 & 42.71 & 89.98 & 5.26 & 5.03 & 3.16 \\
\hline & SE & 1.11 & 0.14 & 0.14 & 0.43 & 0.16 & 1.72 & 0.65 & 0.08 & 0.09 & 0.15 \\
\hline & CD@ $5 \%$ & 3.23 & 0.40 & 0.40 & 1.25 & 0.47 & 5.02 & 1.91 & 0.23 & 0.27 & 0.44 \\
\hline
\end{tabular}


Table.4 Mean value of single cross hybrids for qualitative characters during summer 2017 under IDM condition

\begin{tabular}{|c|c|c|c|c|c|c|c|}
\hline Sl. No. & SCH & $\begin{array}{l}\text { Shelf Life } \\
\text { (days) }\end{array}$ & $\begin{array}{c}\text { Pericarp } \\
\text { Thickness } \\
\text { (mm) }\end{array}$ & $\begin{array}{c}\text { No. of } \\
\text { locules/fruit }\end{array}$ & $\begin{array}{c}\text { Firmness } \\
\left(\mathrm{kg} / \mathrm{cm}^{2}\right)\end{array}$ & $\begin{array}{l}\text { TSS } \\
\left({ }^{\mathbf{0}} \mathrm{B}\right)\end{array}$ & $\begin{array}{l}\text { Lycopene } \\
(\mathrm{mg} / \mathbf{1 0 0 g})\end{array}$ \\
\hline 1. & Arka Rakshak & 25.67 & 6.33 & 3.33 & 5.43 & 5.17 & 6.73 \\
\hline 2. & Arka Samrat & 23.33 & 7.33 & 3.33 & 5.97 & 4.83 & 5.73 \\
\hline 3. & Alankar & 28.00 & 7.33 & 3.00 & 5.87 & 5.17 & 4.73 \\
\hline 4. & Abhinav & 30.33 & 6.67 & 3.00 & 6.90 & 4.83 & 6.93 \\
\hline 5. & 1057 & 32.67 & 7.00 & 3.33 & 5.97 & 4.67 & 5.20 \\
\hline 6. & Badshah & 32.67 & 7.00 & 4.00 & 5.67 & 4.50 & 5.27 \\
\hline 7. & Lakshmi & 30.33 & 7.33 & 3.33 & 5.57 & 5.50 & 5.17 \\
\hline 8. & US3140 & 25.67 & 8.00 & 3.00 & 6.70 & 4.17 & 5.07 \\
\hline 9. & US440 & 25.67 & 6.67 & 3.33 & 6.83 & 4.67 & 6.13 \\
\hline 10. & 1005 & 25.67 & 4.67 & 3.00 & 7.10 & 4.67 & 5.10 \\
\hline 11. & Sonam & 25.67 & 5.67 & 3.33 & 5.33 & 4.00 & 6.70 \\
\hline 12. & Shivam & 30.33 & 6.33 & 3.33 & 7.10 & 4.17 & 5.40 \\
\hline 13. & Ryna & 30.33 & 6.67 & 3.33 & 5.63 & 4.33 & 4.70 \\
\hline \multicolumn{2}{|r|}{ Mean } & 28.18 & 6.69 & 3.28 & 6.16 & 4.67 & 5.61 \\
\hline \multicolumn{2}{|r|}{ SE } & 2.24 & 0.51 & 0.26 & 0.41 & 0.26 & 0.44 \\
\hline \multicolumn{2}{|c|}{ CD@ $9 \%$} & - & 1.50 & - & 1.18 & 0.75 & 1.27 \\
\hline
\end{tabular}


Table.5 Mean value of single cross hybrids for qualitative characters during summer 2017 under Non-IDM condition

\begin{tabular}{|c|c|c|c|c|c|c|c|}
\hline Sl. No. & SCH & $\begin{array}{l}\text { Shelf Life } \\
\text { (days) }\end{array}$ & $\begin{array}{c}\text { Pericarp } \\
\text { Thickness } \\
\text { (mm) }\end{array}$ & $\begin{array}{c}\text { No. of } \\
\text { locules/fruit }\end{array}$ & $\begin{array}{c}\text { Firmness } \\
\left(\mathrm{kg} / \mathrm{cm}^{2}\right)\end{array}$ & $\begin{array}{l}\text { TSS } \\
\left({ }^{0} \mathbf{B}\right)\end{array}$ & $\begin{array}{l}\text { Lycopene } \\
(\mathrm{mg} / \mathbf{1 0 0 g})\end{array}$ \\
\hline 1. & Arka Rakshak & 25.67 & 6.67 & 3.00 & 5.30 & 4.33 & 5.53 \\
\hline 2. & Arka Samrat & 25.67 & 7.33 & 3.33 & 6.27 & 4.33 & 5.73 \\
\hline 3. & Alankar & 28.00 & 6.67 & 3.33 & 5.67 & 4.83 & 4.43 \\
\hline 4. & Abhinav & 25.67 & 6.33 & 3.00 & 7.30 & 6.00 & 5.03 \\
\hline 5. & 1057 & 23.33 & 5.33 & 3.33 & 6.07 & 5.17 & 4.97 \\
\hline 6. & Badshah & 25.67 & 6.00 & 3.67 & 4.73 & 5.00 & 4.53 \\
\hline 7. & Lakshmi & 23.33 & 6.67 & 3.33 & 5.97 & 4.67 & 3.30 \\
\hline 8. & US3140 & 23.33 & 6.00 & 3.33 & 4.87 & 3.00 & 3.67 \\
\hline 9. & US440 & 30.33 & 5.67 & 2.67 & 5.00 & 2.50 & 4.10 \\
\hline 10. & 1005 & 28.00 & 5.67 & 3.33 & 4.57 & 4.33 & 5.40 \\
\hline 11. & Sonam & 28.00 & 5.33 & 3.00 & 5.17 & 3.33 & 6.77 \\
\hline 12. & Shivam & 25.67 & 5.67 & 3.00 & 6.53 & 3.67 & 5.20 \\
\hline 13. & Ryna & 28.00 & 5.67 & 3.00 & 6.60 & 4.33 & 4.10 \\
\hline \multicolumn{2}{|r|}{ Mean } & 26.21 & 6.08 & 3.18 & 5.69 & 4.27 & 4.83 \\
\hline \multicolumn{2}{|r|}{ SE } & 1.94 & 0.38 & 0.26 & 0.37 & 0.34 & 0.47 \\
\hline \multicolumn{2}{|c|}{ CD@ $@ 5 \%$} & - & 1.11 & - & 1.07 & 0.99 & 1.35 \\
\hline
\end{tabular}


Table.6 Performance of top three high yielding single cross hybrids evaluated in field during summer 2017 under IDM and Non-IDM conditions

\begin{tabular}{|c|c|c|c|c|c|c|c|c|c|c|c|c|c|c|c|c|c|c|}
\hline $\begin{array}{l}\text { Sl. } \\
\text { No. }\end{array}$ & SCH & & $\mathbf{P H}$ & D Fl & D Fr & $\mathrm{NC} / \mathrm{P}$ & NFI/C & NFr/P & SFW & FL & FW & $\mathbf{Y}$ & SL & PT & $\mathrm{NL} / \mathrm{P}$ & Fir & TSS & $\mathbf{L y}$ \\
\hline \multirow[t]{2}{*}{1.} & \multirow[t]{2}{*}{ Abhinav } & IDM & 83.87 & 27.73 & 34.13 & 15.07 & 6.00 & 86.33 & 88.87 & 6.28 & 5.23 & 7.46 & 30.33 & 6.67 & 3.00 & 6.90 & 4.83 & 6.93 \\
\hline & & $\begin{array}{l}\text { Non- } \\
\text { IDM }\end{array}$ & 79.60 & 27.60 & 33.47 & 12.47 & 4.93 & 68.73 & 85.60 & 5.89 & 4.93 & 5.79 & 25.67 & 6.33 & 3.00 & 7.30 & 6.00 & 5.03 \\
\hline \multicolumn{3}{|c|}{ Mean } & 81.74 & 27.67 & 33.80 & 13.77 & 5.47 & 77.53 & 87.24 & 6.09 & 5.08 & 6.63 & 28.00 & 6.50 & 3.00 & 7.10 & 5.42 & 5.98 \\
\hline \multirow[t]{2}{*}{2.} & \multirow[t]{2}{*}{ Badshah } & IDM & 114.00 & 27.73 & 34.20 & 16.4 & 5.07 & 70.53 & 85.53 & 5.66 & 4.48 & 5.95 & 32.67 & 7.00 & 4.00 & 5.67 & 4.50 & 5.27 \\
\hline & & $\begin{array}{l}\text { Non- } \\
\text { IDM }\end{array}$ & 105.47 & 28.13 & 34.07 & 13.00 & 3.87 & 54.13 & 85.40 & 5.53 & 4.51 & 4.26 & 25.67 & 6.00 & 3.67 & 4.73 & 5.00 & 4.53 \\
\hline \multicolumn{3}{|c|}{ Mean } & 109.74 & 27.93 & 34.14 & 14.70 & 4.47 & 62.33 & 85.47 & 5.60 & 4.50 & 5.11 & 29.17 & 6.50 & 3.84 & 5.20 & 4.75 & 4.90 \\
\hline \multirow[t]{2}{*}{3.} & \multirow[t]{2}{*}{1057} & IDM & 102.27 & 29.33 & 34.87 & 15.33 & 5.53 & 76.00 & 101.93 & 6.79 & 5.39 & 6.39 & 32.67 & 7.00 & 3.33 & 5.97 & 4.67 & 5.20 \\
\hline & & $\begin{array}{l}\text { Non- } \\
\text { IDM }\end{array}$ & 96.13 & 29.67 & 34.87 & 12.60 & 5.13 & 50.47 & 98.60 & 6.35 & 5.55 & 3.76 & 23.33 & 5.33 & 3.33 & 6.07 & 5.17 & 4.97 \\
\hline \multicolumn{3}{|c|}{ Mean } & 99.20 & 29.50 & 34.87 & 13.97 & 5.33 & 63.24 & 100.27 & 6.57 & 5.47 & 5.08 & 28.00 & 6.17 & 3.33 & 6.02 & 4.92 & 5.09 \\
\hline
\end{tabular}

\begin{tabular}{|c|c|c|c|}
\hline IDM - Integrated Disease Management & $\begin{array}{l}\text { Non-IDM - Non-Integrated Disease } \\
\text { Management }\end{array}$ & PH- Plant height (cm) & D Fl- Days to $1^{\text {st }}$ flower \\
\hline D Fr- Days to $1^{\text {st }}$ fruit & NC/P- Number of clusters/plant & $\mathrm{NFl} / \mathrm{C}-$ Number of flowers/cluster & NFr/P- Number of fruits/plant \\
\hline SFW-Single fruit weight (g) & FL- Fruit length $(\mathrm{cm})$ & FW- Fruit width $(\mathrm{cm})$ & Y- Yield /plant (kg) \\
\hline SL- Shelf Life (days) & PT- Pericarp Thickness (mm) & NL/P- No. of locules/fruit & Fir- Firmness $\left(\mathrm{kg} / \mathrm{cm}^{2}\right)$ \\
\hline TSS- TSS $\left({ }^{0} \mathrm{~B}\right)$ & Ly- Lycopene (mg/100g) & & \\
\hline
\end{tabular}


Table.7 Reaction of single cross hybrids to bacterial wilt in field during summer 2017 under IDM and Non-IDM conditions

\begin{tabular}{|c|c|c|c|c|c|c|}
\hline SI.No. & SCH & $\begin{array}{c}\text { PWI in } \\
\text { IDM }\end{array}$ & $\begin{array}{l}\text { Disease } \\
\text { reaction }\end{array}$ & $\begin{array}{l}\text { PWI in } \\
\text { Non-IDM }\end{array}$ & $\begin{array}{l}\text { Disease } \\
\text { reaction }\end{array}$ & $\begin{array}{c}\text { Over all mean } \\
\text { of PWI }\end{array}$ \\
\hline 1. & Arka Rakshak & 3.12 & $\mathrm{R}$ & 10.41 & $\mathrm{R}$ & 6.77 \\
\hline 2. & Arka Samrat & 6.25 & $\mathrm{R}$ & 15.62 & $\mathrm{R}$ & 10.94 \\
\hline 3. & Alankar & 25 & MR & 46.87 & MS & 35.94 \\
\hline 4. & Abhinav & 0 & $\mathrm{R}$ & 3.12 & $\mathrm{R}$ & 1.56 \\
\hline 5. & 1057 & 1.04 & $\mathrm{R}$ & 15.62 & $\mathrm{R}$ & 8.33 \\
\hline 6. & Badshah & 14.58 & $\mathrm{R}$ & 29.16 & MR & 21.87 \\
\hline 7. & Lakshmi & 20.83 & MR & 25 & MR & 22.92 \\
\hline 8. & US3140 & 4.16 & $\mathrm{R}$ & 9.37 & $\mathrm{R}$ & 6.77 \\
\hline 9. & US440 & 0 & $\mathrm{R}$ & 20.83 & MR & 10.42 \\
\hline 10. & 1005 & 25 & MR & 41.66 & MS & 33.33 \\
\hline 11. & Sonam & 6.25 & $\mathrm{R}$ & 10.41 & $\mathrm{R}$ & 8.33 \\
\hline 12. & Shivam & 6.25 & $\mathrm{R}$ & 22.91 & MR & 14.58 \\
\hline 13. & Ryna & 4.16 & $\mathrm{R}$ & 10.41 & $\mathrm{R}$ & 7.29 \\
\hline
\end{tabular}

PWI- Per cent wilt incidence MS- Moderately susceptible
R- Resistant

S- Susceptible
MR- Moderately resistant 
Table.8 Reaction of single cross hybrids to tomato leaf curl virus in field during summer 2017 under IDM and Non-IDM conditions

\begin{tabular}{|c|c|c|c|c|c|c|c|c|c|c|}
\hline \multirow{2}{*}{$\begin{array}{l}\text { Sl. } \\
\text { No. }\end{array}$} & \multirow[t]{2}{*}{ SCH } & \multicolumn{2}{|c|}{ PDI } & \multicolumn{2}{|c|}{ PDS } & \multirow{2}{*}{$\begin{array}{l}\text { CI } \\
\text { IDM }\end{array}$} & \multirow{2}{*}{$\begin{array}{c}\text { Category } \\
\text { IDM }\end{array}$} & \multirow{2}{*}{$\begin{array}{c}\text { CI } \\
\text { Non-IDM }\end{array}$} & \multirow{2}{*}{$\begin{array}{l}\text { Category } \\
\text { Non-IDM }\end{array}$} & \multirow{2}{*}{$\begin{array}{c}\text { Over all } \\
\text { mean } \\
\text { of CI }\end{array}$} \\
\hline & & IDM & Non-IDM & IDM & Non-IDM & & & & & \\
\hline 1. & $\begin{array}{c}\text { Arka } \\
\text { Rakshak }\end{array}$ & 16.12 & 58.14 & 4.83 & 35.17 & 0.78 & HR & 20.45 & MR & 10.62 \\
\hline 2. & Arka Samrat & 33.33 & 82.71 & 12.77 & 48.45 & 4.25 & $\mathrm{R}$ & 40.08 & S & 22.17 \\
\hline 3. & Alankar & 16.66 & 62.74 & 6.25 & 35.29 & 1.04 & HR & 22.14 & MS & 11.59 \\
\hline 4. & Abhinav & 4.16 & 40.86 & 1.04 & 24.46 & 0.04 & HR & 9.99 & MR & 5.02 \\
\hline 5. & 1057 & 7.36 & 75.30 & 0.13 & 55.24 & 0.13 & HR & 41.60 & S & 20.87 \\
\hline 6. & Badshah & 34.14 & 57.35 & 5.62 & 38.97 & 5.62 & $\mathrm{R}$ & 22.35 & MS & 13.99 \\
\hline 7. & Lakshmi & 23.68 & 100 & 2.02 & 72.91 & 2.02 & HR & 72.91 & HS & 37.47 \\
\hline 8. & US3140 & 15.21 & 55.17 & 0.66 & 40.51 & 0.66 & HR & 22.35 & MS & 11.51 \\
\hline 9. & US440 & 33.33 & 80.26 & 5.12 & 63.15 & 5.12 & $\mathrm{R}$ & 50.69 & $\mathrm{~S}$ & 27.91 \\
\hline 10. & 1005 & 44.44 & 71.42 & 6.94 & 60.26 & 6.94 & $\mathrm{R}$ & 43.04 & $\mathrm{~S}$ & 24.99 \\
\hline 11. & Sonam & 18.88 & 52.32 & 1.15 & 41.57 & 1.15 & HR & 21.75 & MS & 11.45 \\
\hline 12. & Shivam & 11.11 & 72.97 & 0.43 & 56.41 & 0.43 & HR & 41.17 & $\mathrm{~S}$ & 20.80 \\
\hline 13. & Ryna & 19.56 & 75.58 & 1.43 & 56.68 & 1.43 & HR & 42.84 & $\mathrm{~S}$ & 22.14 \\
\hline
\end{tabular}

\begin{tabular}{|c|c|c|c|}
\hline PDI- Per cent disease incidence & PDS- Per cent disease severity & CI- Coefficient of infection & $\begin{array}{l}\text { IDM - Integrated Disease } \\
\text { Management }\end{array}$ \\
\hline $\begin{array}{l}\text { Non-IDM - Non Integrated Disease } \\
\text { Management }\end{array}$ & HR- Highly resistant & R- Resistant & MR- Moderately resistant \\
\hline MS- Moderately susceptible & S- Susceptible & HS- Highly susceptible & \\
\hline
\end{tabular}


In Non-IDM condition: Per cent bacterial wilt incidence ranged from 3.12 to 46.87 per cent. Maximum per cent bacterial wilt incidence was recorded in Alankar (46.87\%) followed by $1005(41.66 \%)$, the minimum per cent bacterial wilt incidence was recorded in Abhinav (3.12\%) followed by US3140 $(9.37 \%)$. For ToLCV, coefficient of infection ranged from 9.99 to 20.45. Maximum coefficient of infection was recorded in Lakshmi (72.91) followed by US440 (50.69), minimum coefficient of infection was recorded in Abhinav (9.99) followed by Arka Rakshak (20.45). The single cross hybrids were classified into four groups based on their reaction to bacterial wilt. Of these, 7 were resistant, 4 were moderately resistant and 2 were moderately susceptible (Table 7). The single cross hybrids were classified into six groups based on their reaction to tomato leaf curl disease. Of these, 2 were moderately resistant, 4 were moderately susceptible, 6 were susceptible and 1 was highly susceptible (Table 8).

Denoyes and Aviers (1989) reported among 25 varieties of tomato evaluated in field for bacterial wilt resistance, 15 were found to be resistant including three hybrids. Four varieties were moderately resistant and six were susceptible. Kapoor et al., (1991) reported that exotic and Indian tomato lines/varieties were screened for resistance to $R$. solanacearum. Of the 62 varieties screened, nine were immune, 26 resistant, five moderately resistant, four moderately susceptible and eighteen were susceptible.

Singh (2014) observed the wild accession, H88-78-1 immune to ToLCV, while the other three genotypes viz. Hissar Lalima, TLBRH-6 and NS-515 were resistant and eight genotypes such as Hissar Anmol, Kashi Vishesh, Kashi Amrit, Kashi Sharad, KS-17, KS-118, Avinash-2 and US-1008 were moderately resistant. Different Lycopersicon accessions with resistance or tolerance to ToLCV are reported by various workers, Lycopersicon esculentum (Mayee et al., 1974 and Varma et al., 1986), L. chilense (Joshi and Choudhari, 1981), L. glandulosum (Varma et al., 1986), L. hirsutum (Joshi and Choudhari, 1981; Banerjee and Kallo, 1987 and Muniyappa et al., 1991a), L. peruvianum (Joshi and Choudhari, 1981 and Banerjee and Kallo, 1987).

Over all mean in both IDM and Non-IDM conditions was worked out for Percent wilt incidence and Coefficient of infection and found Abhinav (1.56\%), US3140 (6.77\%), Arka Rakshak (6.77\%) were top three bacterial wilt resistant single cross hybrids (Table 7) and Abhinav (5.02), Arka Rakshak (10.62), Sonam (11.45) were top three ToLCV resistant single cross hybrids (Table 8).

\section{References}

Arun, J., Amit, V. and Thakur, M. C. 2004. Studies on genetic variability, correlation and path analysis for yield physio-chemical traits in tomato (Lycopersicon esculentum Mill.). Prog. Hort. 36(1): 51-58.

Banerjee, M. K. and Kalloo, G. 1987. Sources of inheritance of resistance to leaf curl virus in Lycopersicon. Theor. Appl. Gen. 73: 707-710.

Bharathkumar, M. V., Sadashiva, A. T. and Jatav, P. K. 2017. Performance of a Set of Tomato Parental Lines and their Hybrids for Quality and Yield under Conditions of Bengaluru, India. Int. J. Curr. Microbiol. App. Sci. 6(5): 786793.

Denoyes, B. and Aviers, A. 1989. Evaluation of tomato materials for use in the processing tomato breeding program in Martinique. In: Green, S. K. ed., Tomato and Pepper Production in the 
Tropics. Shanhua, Tainan, Taiwan, AVRDC, 63-69.

Dheemanth, T. L., Sadhan Kumar, P. G., Nazeem, P. A., Mathew, S, K. and Reddy, M. A. 2017. Field screening of $\mathrm{F} 3$ and F4 generations of tomato for combined resistance to bacterial wilt and tomato leaf curl virus (ToLCV) diseases. Vegetable Science, 44(1): 8185.

Hazarika, T. K. and Phookan, D. B. 2005. Performance of tomato cultivars for polyhouse cultivation during spring summer in Assam. Indian J. Hort. 62(3): 268-271.

Joshi, G. C. and Chaudhary, B. 1981. Screening of Lycopersicon and Solanum species for resistance to leaf curl virus. Veg. Sci. 8: 45-50.

Mayee, C. D., Kanwar, J. S. and Nandapuri, K. S. 1974. The comparative performance of different lines of tomato vis-à-vis leaf curl and mosaic. Punjab Agric. Univ. J. Res. 11: 360-364.

Mew, T. W. and Ho, W. C. 1976. Varietal resistance to bacterial wilt in tomato. $P l$. Disease Reptr. 60: 264-268.

Muniyappa,V., Jalikoppa, S. H., Saikia, A. K., Channarayappa, Shivashankar, G., Ishwarbhat, A. and Ramappa, H. K. 1991a. Reaction of Lycopersicon sp. and wild accessions to tomato leaf curl virus. Euphytica, 56: 37-41.
PDVR. 1997. Resistant varietal trials. In: Proceedings of XVI group meeting on vegetable research, Project Director of Vegetable Research, Varanasi. Pp. 101102.

Renuka, D. M., Sadashiva, A. T., Kavita, B. T., Vijendrakumar, R. C. and Hanumanthiah, M. R. 2014. Evaluation of cherry tomato lines (Solanum lycopersicum Var. cerasiforme) for growth, yield and quality traits. Plant Archives, 14(1): 151-154.

Singh, K. 2014. Evaluation of tomato genotypes and its reaction against ToLCV causing leaf curl disease in tomato (Solanum lycopersicon L.). J. Exp. Biol. Agric. Sci. 2: 120-125.

Varma, J. P., Hayati, J. and Poonam. 1986. Resistance in Lycopersicon species to tomato leaf curl disease in India. Zeitschrift far Pflanzen krankheiteni und pflanzenschtz, 87: 137-144.

Yabuuchi, E., Kosako, Y., Yano, I., Hotta, H. and Nishiuchi, Y. 1995. Transfer of two Burkholderia and an Alcaligenes species to Ralstonia gen. nov. proposal of Ralstonia pickettii (Ralston, Palleroni and Doudoroff, 1973). Comb. nov., Ralstonia solanacearum (Smith, 1896) Comb nov. and Ralstonia eutropha (Davis, 1969) Comb. Nov. Microbiol. Immunol. 39: 897-904.

\section{How to cite this article:}

Dheemanth, T. L., B. G. Prakash, M. K. Honnabyraiah, A. P. Mallikarjun Gowda and Mohan Kumar, S. 2020. Evaluation of single cross hybrids in tomato (Solanum lycopersicum) under IDM and Non-IDM conditions for resistance to bacterial wilt and tomato leaf curl virus diseases. Int.J.Curr.Microbiol.App.Sci. 9(02): 2697-2710.

doi: https://doi.org/10.20546/ijcmas.2020.902.307 